\title{
Evolution of the Human Cytokine Response from Acute Illness to Disease Resolution in SARS-Cov-2 Infection-Implications for Therapeutic Monitoring and Therapeutic Targets
}

\author{
George P. Drewett ${ }^{1,2}$ (D) Ana Copaescu ${ }^{2,3} \cdot$ Effie Mouhtouris $^{1,2} \cdot$ Natalie Hannan $^{4} \cdot$ Fiona James $^{1,2} \cdot$ Olivia Smibert $^{1}$. \\ Natasha E. Holmes ${ }^{1,2,5,8}$ • Jason A. Trubiano ${ }^{1,2,5,6,7}$
}

Received: 28 December 2020 / Accepted: 8 April 2021 / Published online: 16 April 2021

(C) The Author(s), under exclusive licence to Springer Science+Business Media, LLC, part of Springer Nature 2021

\section{To the Editor:}

There has been considerable interest in the role of cytokines in acute COVID-19 as markers of disease severity, monitoring response to therapy, and as potential targets for therapeutic intervention [1]. The ad-hoc nature of patient sampling and use of single cytokine targets (e.g., IL-6) have limited our understanding of COVID-19 immune responses. We used a unique, prospective COVID-19 cohort with timed patient sampling to explore longitudinal, comprehensive, cytokine responses from hospital admission (acute illness) to discharge (recovery).

\section{Methods}

We performed a prospective observational cohort study of adult patients admitted with COVID-19 between May and October 2020 at Austin Health, Melbourne, Australia. Baseline demographic and laboratory data were collected. Plasma was collected at time of hospital admission and discharge. The patient sampling procedure is outlined in Supplementary Fig. 1. Inpatient management, including decisions regarding commencement

George P. Drewett

gdrewett@gmail.com; George.drewett@austin.org.au

1 Department of Infectious Diseases, Austin Health, Heidelberg, Victoria, Australia

2 Centre for Antibiotic Allergy and Research, Department of Infectious Diseases, Austin Health, Heidelberg, Victoria, Australia

3 Department of Clinical Immunology and Allergy, McGill University Health Center, Montréal, Canada of corticosteroid and remdesivir therapies, was directed by infectious diseases physicians, based on local protocols.

Cytokine analysis was performed on plasma using a multiplex bead array kit 17-plex panel (BioPlex Pro Human cytokine 17 plex assay-M5000031YV, Bio-Rad), and read using the BioPlex 200 instrument (Bio-Rad) as per manufacturer's instructions. Cytokine levels below the lower limit of detection of the assay were recorded as $0 \mathrm{pg} / \mathrm{mL}$.

Statistical analysis was performed using Stata MP 16.1 (StataCorp, College Station, TX). Wilcoxon ranksum test was used for univariate analysis across timepoints. Exploratory subgroup and multivariable analyses were performed but limited by the effect of small sample size on statistical power. Subgroups compared were Intensive Care Unit (ICU) admission and receipt of corticosteroids, and multiple linear regression was performed for the change in key cytokine levels with selected clinically relevant variables (Charlson comorbidity index [CCI], ICU admission, receipt of corticosteroids). Ethics approval was granted at Austin Health (HREC/63201/Austin-2020).

4

Therapeutics Discovery and Vascular Function Laboratory, Translational Obstetrics Group, Department of Obstetrics \& Gynaecology, The University of Melbourne, Heidelberg, Victoria, Australia

5 Department of Medicine (Austin Health), University of Melbourne, Heidelberg, Victoria, Australia

6 Department of Oncology, Sir Peter MacCallum Cancer Centre, The University of Melbourne, Parkville, Victoria, Australia

7 The National Centre for Infections in Cancer, Peter MacCallum Cancer Centre, Parkville, Victoria, Australia

8 Department of Medicine and Radiology, Melbourne Medical School, The University of Melbourne, Parkville, Victoria, Australia 


\section{Results}

Seventy-eight participants were enrolled in the study and had admission plasma collected, of whom 71 had admission plasma analyzed via the multiplex assay, and 35 had matching plasma collected and analyzed at discharge and are included in this analysis. The results of the paired multiplex assay $(N=$ 35) are summarized in Supplementary Table 1. For the final cohort of 35 matching patients, the median age-adjusted CCI was 2 (IQR 1, 4), median length of stay was 6 days (IQR 313), $22(63 \%)$ patients received corticosteroid therapy (6 mg oral dexamethasone daily for 5 days), and 17 (49\%) required supplementary oxygen. Median admission $\mathrm{PaO} 2 \mathrm{FiO} 2$ ratio was 376, and $11(31 \%)$ patients required intensive care admission. Additional demographic, laboratory, and treatment data can be found in Supplementary Table 2. Multiplex assay results for all 71 patients at single admission timepoint can be found in Supplementary Table 3.

From admission to discharge, a significant decrease in interleukin (IL)-6 (5.05 vs $0 \mathrm{pg} / \mathrm{mL}, p<0.001)$, tumor necrosis factor alpha (TNF-a) (9.62 vs $6.17 \mathrm{pg} / \mathrm{mL}, p=0.002)$, monocyte chemoattractant protein-1 (MCP-1) $(9.59 \mathrm{vs} 4.26 \mathrm{pg} / \mathrm{mL}$, $p=0.02$ ) was observed (Fig. 1). A significant difference in IL$1 \mathrm{~b}(p=0.03)$ was also detected, although levels at admission and discharge were both very low.

Detectable levels of IL-7, IL-8, and IL-10 were found, although these had not significantly decreased at time of discharge. Levels of IL-2, IL-5, IL-12, IL-13, IL-17, granulocyte colony stimulating factor (G-CSF), and granulocyte macrophage colony stimulating factor (GM-CSF) were not elevated at either timepoint.

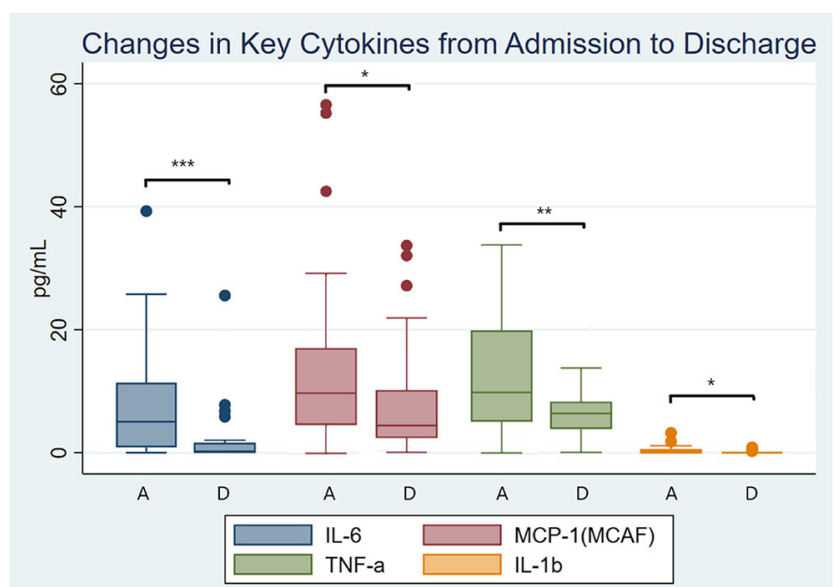

Fig. 1 Changes in key cytokines from admission to discharge. A: admission, D: discharge, IL: interleukin, MCP-1: monocyte chemoattractant protein-1, TNF-a: tumor necrosis factor alpha. $* * * p<0.001, * * p<0.01, * p<0.05$. Outlier values are marked with points, all other data points lie within the box plots. All cytokines shown in the figure demonstrated statistically significant reductions (see Supplementary Table 1)
There were no statistically significant differences between subgroups of ICU admission and receipt of corticosteroids (Supplementary Tables 5 and 6). Multiple linear regression for the change in the four key cytokines identified in Fig. 1 between admission and discharge and CCI, ICU admission, and receipt of corticosteroids is shown in Supplementary Table 7 and also revealed no statistically significant differences.

\section{Discussion}

Our study has simultaneously compared the dynamics of SARS-CoV-2 infection across 17 cytokines over the course of patients' hospital admissions. It is notable that the majority of cytokines measured were not increased at either timepoint, suggesting a lesser potential role for interventions that target these pathways and for therapeutic monitoring.

IL-6 has frequently been implicated in the inflammatory cascade associated with severe COVID-19 [1, 2], as have IL-1b and TNF-a [3, 4]. Elevated levels of TNF-a and MCP1 have also been demonstrated in SARS-CoV-2 infected ACE2+ pneumocytes [5]. The significant dynamic reduction in these cytokines over time provides evidence to implicate these molecules in the inflammatory state associated with moderate and severe COVID-19 infection, and that the reduction in expression of these molecules is associated with clinical resolution.

Although COVID-19 is often a highly inflammatory process, our study found only a few cytokines actually elevated in hospitalized patients. There is, however, a definite change over time in several cytokines that correlates with clinical recovery, suggesting a potential role for these cytokines as biomarkers, and providing a biologically plausible explanation for the reported efficacy of therapeutic agents targeting these cytokines such as the IL- 6 inhibitors, tocilizumab and sarilumab [6].

Our study is limited by its small sample size, due to the to-date relatively low prevalence of COVID-19 in our setting. We postulate that this may explain the absence of significant differences between subgroups such as those requiring ICU admission or receipt of steroids, due to insufficient statistical power. Given the interplay between corticosteroid therapy and the immune response, and speculative correlation between COVID-19 severity and cytokine response, it would be beneficial to perform these analyses on a larger cohort to further explore these interactions. A number of cytokines with potential roles in the immune response to Sars-CoV-2 infection, such as type 1 interferons, were not included in this analysis as they were not measurable using the Bioplex panel. Furthermore, this study has only included admitted patients. As such, the results may not be generalizable to the full spectrum of 
patients with COVID-19 disease, particularly to outpatients with less severe disease. Nonetheless, this study examines a prospectively collected cohort with matching analysis at time of illness presentation and convalesce.

\section{Conclusion}

Our immune COVID-19 cytokine study, which longitudinally measured 17 common cytokines at paired admission and discharge timepoints for 35 patients hospitalized with COVID19 , found an elevation in several clinically significant cytokines, and a significant reduction in IL-6, TNF-a, IL-1b, and MCP-1 associated with disease convalescence, adding further weight to the immunopathological importance of these cytokines in the development and resolution of COVID-19 and future pathways for disease modulation.

Supplementary Information The online version contains supplementary material available at https://doi.org/10.1007/s10875-021-01039-x.

Author Contributions All authors contributed to this work.

Funding This study was supported by the Austin Health Fundraising.

Data Availability All data (de-identified) is stored on secure databases at Austin Health and is available on request.

\section{Declarations}

Ethics Approval This study was approved by the Austin Health Human Research Ethics Committee. Approval number: HREC/63201/Austin2020 .
Consent to Participate Participants provided written consent to participate in this study.

Consent for Publication The authors consent to this work being published.

Competing Interests The authors declare no competing interests.

\section{References}

1. Copaescu A, Smibert O, Gibson A, Phillips EJ, Trubiano JA. The role of IL-6 and other mediators in the cytokine storm associated with SARSCoV-2 infection. J Allergy Clin Immunol. 2020;146(3):518-34 e1.

2. Gustine JN, Jones D. Immunopathology of hyperinflammation in COVID-19. Am J Pathol. 2021;191(1):4-17.

3. Del Valle DM, Kim-Schulze S, Huang HH, Beckmann ND, Nirenberg $\mathrm{S}$, Wang B, et al. An inflammatory cytokine signature predicts COVID19 severity and survival. Nat Med. 2020;26(10):1636-43.

4. Chen G, Wu D, Guo W, Cao Y, Huang D, Wang H, et al. Clinical and immunological features of severe and moderate coronavirus disease 2019. J Clin Invest. 2020;130(5):2620-9.

5. He L, Ding Y, Zhang Q, Che X, He Y, Shen H, et al. Expression of elevated levels of pro-inflammatory cytokines in SARS-CoVinfected ACE2+ cells in SARS patients: relation to the acute lung injury and pathogenesis of SARS. J Pathol. 2006;210(3):288-97.

6. REMAP-CAP Investigators, Gordon AC, Mouncey PR, et al. Interleukin-6 Receptor Antagonists in Critically Ill Patients with Covid-19. N Engl J Med. 2021 Feb 25

Publisher's Note Springer Nature remains neutral with regard to jurisdictional claims in published maps and institutional affiliations. 